\title{
Design of low power pipelined ADC
}

\author{
Amit Maheshwari ${ }^{1}$, Gaurav Gupta ${ }^{2}$ \\ Student, Electronics \& Communication Department, MIT Ujjain, M.P. (India) ${ }^{1}$ \\ Assistant Professor, Electronics \& Communication Department, MIT Ujjain, M.P. (India) ${ }^{2}$
}

\begin{abstract}
A design of 8 bits, $2.5 \mathrm{~V}$ pipeline ADC is introduced in this paper. The comparator is the main improvement aiming at realizing low power dissipation. The latched comparator is adopted to achieve the specification. The design is implemented under 0.25 um CMOS technology which achieves a power dissipation of $205.9 \mathrm{~mW}$.
\end{abstract}

Keywords: Comparator, Op-amp, SUB-ADC, MDAC, SNDR, ENOB

\section{INTRODUCTION}

Pipelined analog-to-digital(ADC)architecture has gained further amplified by the positive feedback action of the great popularity in data communication and video latch as the comparator goes into latch mode. The latch processing applications where high sampling rates and output is then applied to inverters sized in such a way as to medium-to-high resolutions are necessary Due to the lower the threshold point to prevent errors of comparator increasing demand for portability in these applications, [4].Outputs of ADC remains 0 until the comparator has reducing the power consumption of ADC has become one sufficient overdrive voltage to cause one output to toggle of the key design criteria. While the pipelined ADC high. For resolution lower than 3 bits, dynamic architecture is well suited for high sampling rates, comparators can be used for the inter-stage ADCs since reducing the power consumption. The proposed they do not consume de power. ADC will need to be architecture consists by means of 4 separate 2 bit ADC preceded by continuous time preamplifiers to reduce the sub-blocks, 1 MDAC block and 1 SHA block

\section{PIPELINED ADC}

Pipeline ADCs are also high speed ADCs and can be capable of resolving medium to high resolutions. [1] These ADCs work by converting a signal from analog to digital in stages. Each stage converts a portion of the output resolution. The first stage converts the most significant bits (MSB) and the subsequent stages convert less significant bits until the least significant bits (LSB) are converted. The overall general architecture of a pipeline ADC is shown in Figure 1. Each stage has a similar structure, shown exploded in Figure1. Each block contains a sample and hold block to sample the analog signal. This feeds into a small flash converter that resolves n-bits. This n-bit output is fed back through a DAC and the binary value is subtracted from the original input signal to generate a residue voltage.

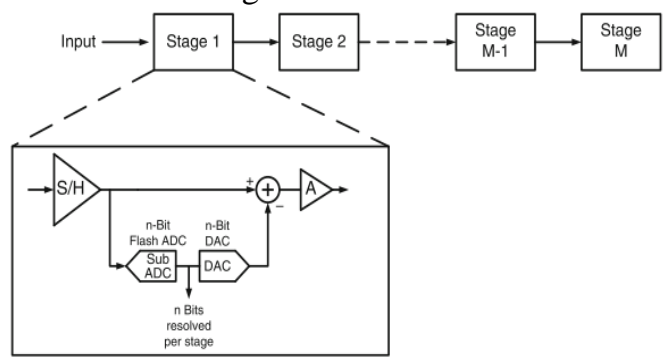

Figure1: Pipelined ADC

\section{PROPOSED DESIGNS}

\section{A) Proposed Comparator}

For the pipelined ADCs latched comparator is used. When $\phi_{\text {latch }}$ goes high 1, the gain transistors gets turn on, it amplifies the differential signal at the inputs $V_{\text {in }}$ which is

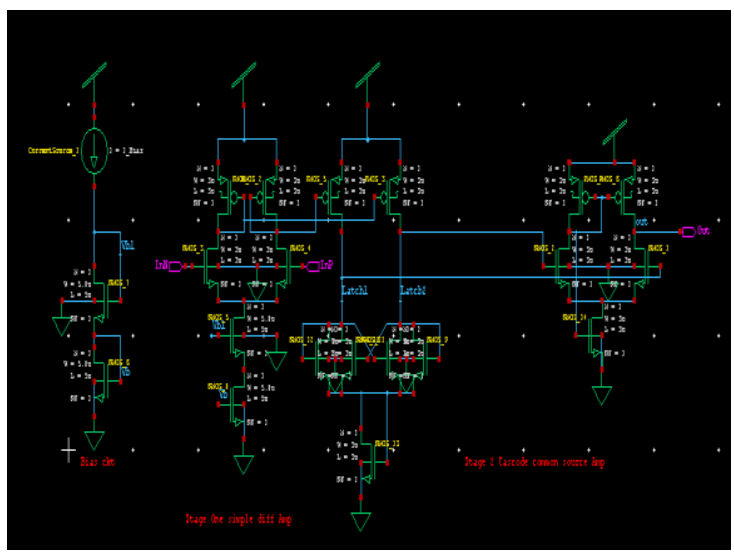

Figure 2: Schematic of comparator

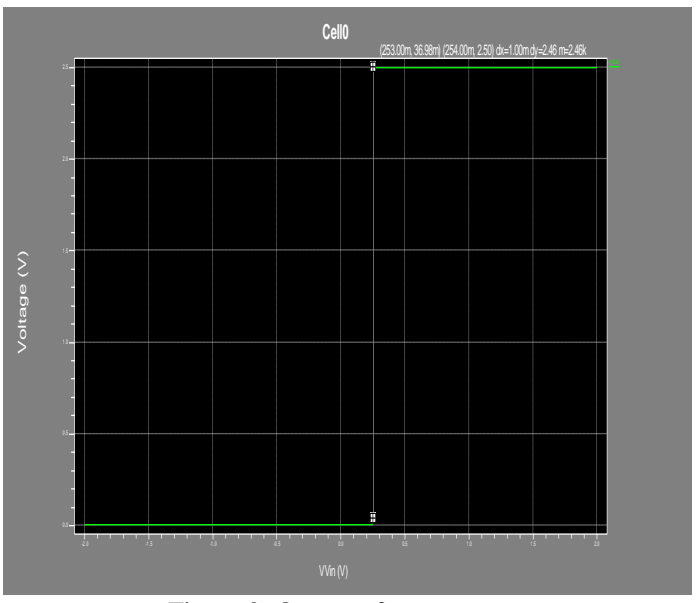

Figure 3: Output of comparator 
INTERNATIONAL JOURNAL OF INNOVATIVE RESEARCH IN ELECTRICAL, ELECTRONICS, INSTRUMENTATION AND CONTROL ENGINEERING Vol. 2, Issue 12, December 2014

Table I:

Various parameters and their values

\begin{tabular}{|c|c|c|}
\hline S. No. & Parameter & Value \\
\hline 1 & DC gain & 2372 \\
\hline 2 & Offset Voltage & $1.38 \mathrm{~mW}$ \\
\hline 3 & Power Dissipation & $4.023 \mu \mathrm{W}$ \\
\hline
\end{tabular}

The design implementation of comparator consists of a latch stage preceded by coupling capacitors that are precharge to the input signal and reference voltages during the reset phase 4.023 microwatt dc power is dissipating in this comparator.

\section{B) Proposed MDAC}

MDAC (multiplying digital to anlog converters) commonly used in pipelined ADC's . Digital calibration technique is implemented in MDAC.Figure 4 shows the dsign of Multiplying digital to analog converter.

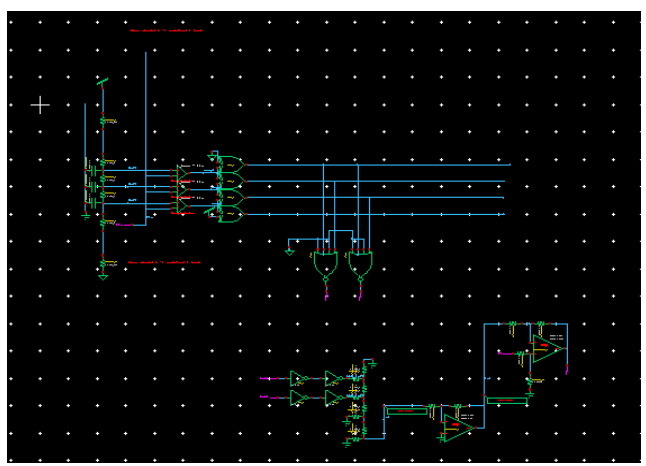

Various parameters and their values

\begin{tabular}{|c|c|c|}
\hline S.No. & Parameter & Value \\
\hline 1 & AC Gain & $81.52 \mathrm{~dB}$ \\
\hline 2 & Phase Margin & $69^{\circ}$ \\
\hline 3 & Unity Gain Bandwidth product & $162.61 \mathrm{MHz}$ \\
\hline 4 & Power dissipation & $8.507 \mathrm{~mW}$ \\
\hline
\end{tabular}

\section{CONCLUSION}

This work presents a novel front end technique that significantly reduces the power consumption in the front end $\mathrm{S} / \mathrm{H}$. The goal of this dissertation is to design a low power consumption ADC .The goal is accomplished by designing a $2.5 \mathrm{~V}, 2$ bit/stage 8-bit ADC by using Latched Comparator in MDAC. The ADC is designed in $0.25 \mu \mathrm{m}$ CMOS process at $2.5 \mathrm{~V}$ supply. At an input frequency of 1 $\mathrm{MHz}$ and a sampling rate of $5 \mathrm{MS} / \mathrm{s}$, it consumes a power of $205.9 \mathrm{~mW}$ with 39.41 dB SNDR, 6.25 ENOB, 2.3/-2.1 LSB INL and +0.85/-0.21 LSB DNL. Performance for proposed design has been summarized in tabular form shown in below table.

The op-amp is used in the MDAC to implement the stage gain. It's configured in a closed-loop circuit where the closed-loop gain is equal to the stage gain. The three opamp design parameters that are discussed next are gain, bandwidth, and output swing.

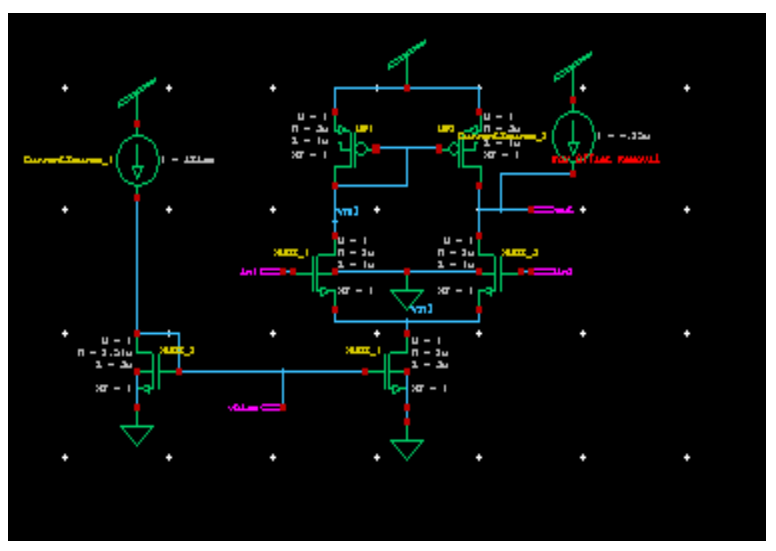

Figure 5: Cascode Op-amp

Table III: Performance summary

\begin{tabular}{|c|c|}
\hline Technology & $0.25 \mu \mathrm{m}$ CMOS process \\
\hline Resolution & $8 \mathrm{bit}$ \\
\hline Supply Voltage & $2.5 \mathrm{~V}$ \\
\hline Conversion rate & $5 \mathrm{MS} / \mathrm{s}$ \\
\hline SNDR & $39.41 \mathrm{~dB}$ \\
\hline ENOB & 6.25 \\
\hline INL/DNL & $+2.3 /-2.1 \mathrm{LSB} /+0.85 /-0.21$ \\
$\mathrm{LSB}$ \\
\hline Power & $205.9 \mathrm{~mW}$ \\
\hline
\end{tabular}

\section{REFERENCES}

[1] B.G.Lee , B.M.Min, "A 14-bit $100 \mathrm{MS} / \mathrm{s}$ Pipeline ADC with a Merged SHA and First MDAC", IEEE Journal of solid state circuits, Vol.24, No. 12, December 2008.

[2] B.G. Lee, R.M Tsang, "A 10-bit 50 MS/s Pipelined ADC With Capacitor-Sharing and Variable- $\mathrm{g}_{\mathrm{m}}$ Op-amp", IEEE Journal of solid state circuits, Vol. 44, No. 3, March 2009.

[3] N. Sasidhar, Y.J. Kook, "A Low Power Pipeline ADC Using Capacitor and Opamp Sharing Technique With a Scheme to Cancel the Effect of Signal Dependent Kickback", IEEE Journal of solid state circuits, Vol. 44, No. 9, September 2009. 
INTERNATIONAL JOURNAL OF INNOVATIVE RESEARCH IN ELECTRICAL, ELECTRONICS, INSTRUMENTATION AND CONTROL ENGINEERING Vol. 2, Issue 12, December 2014

[4] D. Kurose, T. Ito, "55-mW 200-MSPS 10-bit Pipeline ADCs for Wireless Receivers", IEEE Journal of solid state circuits, Vol.41, No. 7, July 2006.

[5] S.T. Ryu, B.S. Song, "A 10-bit 50-MS/s Pipeline ADC With Opamp Current Reuse", IEEE Journal of solid state circuits, Vol.42, No. 3, March 2007.

[6] K. Honda, M. Furuta, "A Low-Power Low-Voltage 10-bit 100MSample/s Pipeline A/D Converter Using Capacitance Coupling Techniques", . IEEE Journal of solid state circuits, Vol.24, No. 4, December 2007.

[7] A. Verma, B. Razavi, "A 10-Bit 500-MS/s 55-mW CMOS ADC", IEEE Journal of solid state circuits, Vol. 44, No. 11, November 2009.

[8] C.S Shin, G.C. Ahn, "A 10-bit 100-MS/s Dual-Channel Pipeline ADC Using Dynamic Memory Effect Cancellation technique", IEEE Transactions on circuit and system, Express Briefs, Vol. 58, No. 5, May 2011.

[9] C.J. Tseng, H.W. Chen, “A 10-bit 320-MS/s Stage-Gain-Error SelfCalibration Pipeline ADC", IEEE Journal of solid state circuits, Vol. 47, No. 6, June 2012

[10] S. Devarajan, L. Singer, "A 16-bit, $125 \mathrm{MS} / \mathrm{s}, 385 \mathrm{~mW}, 78.7 \mathrm{~dB}$ SNR CMOS Pipeline ADC", IEEE Journal of solid state circuits, Vol. 44, No. 12, December 2009.

[11] J. Yuan, S.W. Fung, "An Interpolation-Based Calibration Architecture for Pipeline ADC With Nonlinear Error", IEEE Transactions on instrumentation and measurement, Vol.61, No.1, January 2012

[12] T. Sundstrom, C. Svensson, "A 2.4 GS/s, Single-Channel, 31.3 dB SNDR at Nyquist, Pipeline ADC in $65 \mathrm{~nm}$ CMOS", IEEE Journal of solid state circuits, Vol. 46, No. 7, July 2011.

[13] C.y. Chen, J. Wu, "A 12-Bit 3 GS/s Pipeline ADC With $0.4 \mathrm{~mm}^{2}$ and $500 \mathrm{~mW}$ in $40 \mathrm{~nm}$ Digital CMOS", IEEE Journal of solid state circuits, Vol. 47, No. 4, April 2012. 